\title{
Manipulating and squeezing the photon local density of states with plasmonic nanoparticle networks
}

\author{
Christian Girard, Erik Dujardin, Renaud Marty, and Arnaud Arbouet \\ CEMES, CNRS-Université Paul Sabatier, 29 rue J. Marvig, F-31055 Toulouse, France \\ Gérard Colas des Francs \\ Laboratoire Interdisciplinaire Carnot de Bourgogne, UMR 5209, CNRS-Université de Bourgogne, 9 Avenue A. Savary, BP 47870, \\ F-21078 Dijon, France
}

(Received 2 February 2010; revised manuscript received 15 March 2010; published 27 April 2010)

\begin{abstract}
In this Brief Report, we show that when interconnected networks of gold particles are deposited onto a clean planar surface, they strongly modify the photonic local density of states (LDOS) in the immediate proximity of the self-assembled nanoparticles. They represent unique architectures for the subwavelength patterning of initially flat photonic LDOS. Moreover, we show that their local spectral signatures are well suited for the generation of sites able to enhance molecular fluorescence intensity.
\end{abstract}

DOI: 10.1103/PhysRevB.81.153412

PACS number(s): 78.20.Bh, 73.20.Mf, 68.37.Uv

Recently, the fundamental limitations of light confinement due to diffraction have been significantly reduced with the advent of high refractive-index materials ${ }^{1,2}$ or the tailoring of surface plasmon modes. ${ }^{3-9}$ This light confinement clearly appears when imaging the optical local density of states (LDOS) in the vicinity of the nanostructures in the direct space $(X Y Z) .{ }^{10-12}$ Scanning near-field optical microscopes (SNOM) deliver optical signals proportional to the LDOS at the successive positions of the tip. ${ }^{13}$ Furthermore, in the case of optical corrals, LDOS maps reveal standing optical states confined inside closed patterns such as circles, stadiums, rings, or ellipses microfabricated at the surface of dielectric materials. ${ }^{14,15}$ In experiments involving micron-sized lithographically designed structures, the LDOS intensity can be tuned and enhanced at the corrals center. ${ }^{14,15}$ The image is then dominated by pseudoperiodic ripples (ringlike modes) that reveal the nonevanescent states lying around and inside the corrals.

Although electromagnetic field enhancement and confinement have been extensively studied with lithographically designed nanostructures, the actual spatial distribution of the LDOS and the role of surface-plasmon modes in much smaller colloidal nanoparticles assemblies remain broadly unexplored. Some indications were recently gathered by Imura et al. ${ }^{12,16}$ who performed near-field optical imaging of plasmon modes in single gold nanorods. An apertured SNOM setup was used to excite two-photon-induced photoluminescence on single nanorods. Photoluminescence spectra show several optical resonances that are attributed to different surface-plasmon modes, and according to numerical analysis, their intensities are related to the optical LDOS.

In this context, the simple fabrication of complex and extended networks of interconnected chains of nanoparticles by a spontaneous self-assembly process ${ }^{17-19}$ has been reported (cf. Fig. 1). The optical properties of these superstructures, that we called plasmonic nanoparticles networks (PNN) can be probed experimentally and modeled by the discrete dipole approximation (DDA) numerical scheme. ${ }^{17}$ In particular, the longitudinal mode can be seen as resulting from the efficient coupling of nanoparticle surface plasmons by dipolar interactions between neighboring but nontouching nanoparticles. The transfer of colloidal particles from a suspension onto a solid substrate has been a major challenge in recent years for its importance in photonics, for example, in the fabrication of photonic band-gap devices by depositing a few layers of colloidal photonic crystal ${ }^{20}$ or in using deposited colloids as masks for shadow evaporation (nanosphere lithography) of plasmonic metal nanopatterns. ${ }^{21}$ However, the controlled deposition, spreading and addressing of the self-assembled colloidal superstructures still remain challenging. Very recently, by systematically controlling the surface chemistry of the substrate, the deposition method and the drying regime of the liquid colloidal solution, we have shown that it is possible to deposit intact and well-spread networks. ${ }^{22}$ Near a planar dielectric surface, the optical properties of such objects lead to subwavelength patterning of initially flat optical near fields. ${ }^{22}$

In this Brief Report, we develop an efficient scheme able to compute the photon LDOS generated by complex PNNs or many other colloidal self-assemblies. In a second step, we

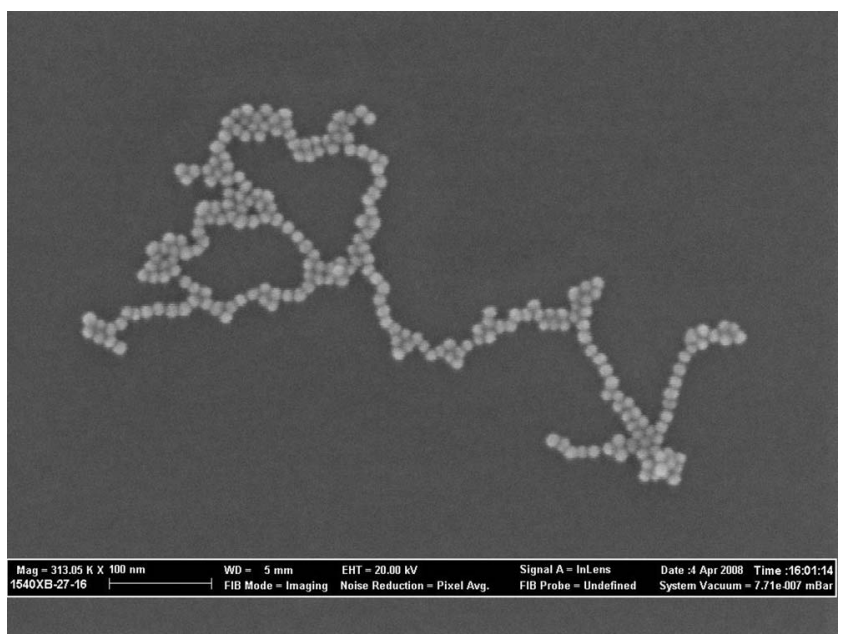

FIG. 1. Transmission electron microscopy (TEM) image showing a self-assembled Au nanoparticle chain network deposited on a glass substrate (see Ref. 22). 
explore the LDOS maps and spectra generated by metallic particle chains and PNNs by applying a self-consistent DDA approach. ${ }^{22,23}$ Since the role of the LDOS in the decay rate process of fluorescent molecules is well identified in optics, ${ }^{24}$ we will exploit it to show that the sub-50-nm-scale patterning of the LDOS by PNN can be used to spatially engineer the molecular fluorescence intensity with unprecedented resolution.

In a perfectly homogeneous medium which sustains only radiative modes, the three usual Cartesian directions are equivalent so that this quantity $\rho(\mathbf{r}, \omega)$ appears as a sum of three equivalent quantities $\rho_{\alpha}(\mathbf{r}, \omega)$ (with $\alpha=x, y$, or $z$ ) that represent the three polarized LDOS (also called partial LDOS). This property is no longer satisfied in the presence of a corrugated surface or a surface supporting small particles. For instance, in the vicinity of dielectric particles adsorbed on a surface, the three polarized LDOS $\rho_{\alpha}(\mathbf{r}, \omega)$ (with $\alpha=x, y$, or $z$ ) are dramatically modified by the presence of the evanescent modes sustained by the particles. ${ }^{25}$ Additionally, in this case where no significant energy dissipation is expected inside the material, each polarized $\operatorname{LDOS} \rho_{\alpha}(\mathbf{r}, \omega)$ is strictly proportional to the imaginary part of the Green'sfunction component $\mathcal{S}_{\alpha, \alpha}\left(\mathbf{r}, \mathbf{r}^{\prime}, \omega\right)$,

$$
\rho_{\alpha}(\mathbf{r}, \omega)=\frac{1}{2 \pi^{2} \omega} \operatorname{Im}\left[\mathcal{S}_{\alpha, \alpha}(\mathbf{r}, \mathbf{r}, \omega)\right]
$$

The detailed demonstration of this relation (1) is provided in reference. ${ }^{10}$ We should point out that Eq. (1) does not represent stricto sensu the local density of states of plasmonic structures which generally exhibit significant dissipative properties because nonradiative decay channels introduce additional contributions. Nevertheless, this relation remains valid to study the fluorescence properties of molecules located near plasmonic components because it provides the total decay rate or to analyze the electron-energy loss spectroscopy signals generated by fast electrons impinging a metal particle. ${ }^{26,27}$ To apply this relation to a PNN structure containing $N$ gold nanoparticles located at the positions $\mathbf{r}_{i}$, we express the Green dyadic function $\mathcal{S}\left(\mathbf{r}, \mathbf{r}^{\prime}, \omega\right)$ in term of the individual polarizabilities $\alpha_{j}(\omega)(j=1-N)$ associated with each nanoparticle that composes the network,

$$
\mathcal{S}\left(\mathbf{r}, \mathbf{r}^{\prime}, \omega\right)=\mathbf{S}^{(o)}\left(\mathbf{r}, \mathbf{r}^{\prime}, \omega\right)+\sum_{j=1}^{N} \alpha_{j}(\omega) \mathbf{S}^{(o)}\left(\mathbf{r}, \mathbf{r}_{j}, \omega\right) \cdot \mathcal{S}\left(\mathbf{r}_{j}, \mathbf{r}^{\prime}, \omega\right),
$$

where $\mathbf{S}^{(o)}\left(\mathbf{r}, \mathbf{r}^{\prime}, \omega\right)$ represents the Green dyadic function of the bare sample surface (i.e., in the absence of any PNN). Analytical expressions of this field susceptibility can be found in the literature [see, for example, Eqs. (35) and (37) of Ref. 28]. For large number $N$ of plasmonic particles, this self-consistent equation must be solved numerically (for example, by applying the Dyson sequence algorithm or by applying a simple matrix inversion method). However analytical expressions can be obtained when considering single or isolated particles adsorbed on plane surfaces. According to Eq. (2), a single spherical nanoparticle, located at $\mathbf{R}$
$=(0,0, R)$, modifies the field susceptibility $\mathcal{S}\left(\mathbf{r}, \mathbf{r}^{\prime}, \omega\right)$ of the bare surface, which now reads,

$$
\mathcal{S}\left(\mathbf{r}, \mathbf{r}^{\prime}, \omega\right)=\mathbf{S}^{(o)}\left(\mathbf{r}, \mathbf{r}^{\prime}, \omega\right)+\alpha(\omega) \mathbf{S}^{(o)}(\mathbf{r}, \mathbf{R}, \omega) \cdot \mathcal{S}\left(\mathbf{R}, \mathbf{r}^{\prime}, \omega\right) .
$$

To solve this equation, we first determine the tensor $\mathcal{S}\left(\mathbf{R}, \mathbf{r}^{\prime}, \omega\right)$ by putting $\mathbf{r}=\mathbf{R}$ in Eq. (3),

$$
\mathcal{S}\left(\mathbf{R}, \mathbf{r}^{\prime}, \omega\right)=\mathbf{A}^{-1}(\omega, \mathbf{R}) \cdot \mathbf{S}^{(o)}\left(\mathbf{R}, \mathbf{r}^{\prime}, \omega\right),
$$

where $\mathbf{A}(\omega, \mathbf{R})$ is a simple diagonal $3 \times 3$ matrix built from the dipolar polarizability, the identity matrix $\mathbf{I}$, and the diagonal tensor $\mathbf{S}^{(o)}(\mathbf{R}, \mathbf{R}, \omega)$,

$$
\mathbf{A}(\omega, \mathbf{R})=\left[\mathbf{I}-\alpha(\omega) \mathbf{S}^{(o)}(\mathbf{R}, \mathbf{R}, \omega)\right] .
$$

This procedure leads to the analytical result,

$$
\begin{aligned}
\mathcal{S}\left(\mathbf{r}, \mathbf{r}^{\prime}, \omega\right)= & \mathbf{S}^{(o)}\left(\mathbf{r}, \mathbf{r}^{\prime}, \omega\right)+\alpha(\omega) \mathbf{S}^{(o)}(\mathbf{r}, \mathbf{R}, \omega) \cdot \mathbf{A}^{-1}(\omega, \mathbf{R}) \\
& \cdot \mathbf{S}^{(o)}\left(\mathbf{R}, \mathbf{r}^{\prime}, \omega\right) .
\end{aligned}
$$

Finally, substitution of Eq. (6) into relation (1) yields the three polarized LDOS's $\rho_{\alpha}^{s}(\mathbf{r}, \omega)$ generated by a single nanoparticle. The total LDOS then reads,

$$
\begin{aligned}
\rho^{\text {single }}(\mathbf{r}, \omega)= & \bar{\rho}(z, \omega)+\frac{1}{2 \pi^{2} \omega} \operatorname{Im}\{\alpha(\omega) \\
& \left.\times \operatorname{Tr}\left[\mathbf{S}^{(o)}(\mathbf{r}, \mathbf{R}, \omega) \cdot \mathbf{A}^{-1}(\omega, \mathbf{R}) \cdot \mathbf{S}^{(o)}(\mathbf{R}, \mathbf{r}, \omega)\right]\right\},
\end{aligned}
$$

where the quantity $\bar{\rho}(z, \omega)$, invariant in all $(X O Y)$ planes, defines the total LDOS in front of the bare glass surface. In addition, the symbol Tr means that the trace of the tensorial quantity between brackets must be taken. From relation (7), we have modeled LDOS maps in the vicinity of noninteracting gold nanoparticles deposited on a glass surface (Fig. 2). In the three maps, a distance $Z_{0}=60 \mathrm{~nm}$ separates the particle centers from the observation plane. In the Fig. 2(B), the normalized total LDOS $\rho^{\text {single }}(\mathbf{r}, \omega) / \bar{\rho}(z, \omega)$ displays symmetrical protrusions localized around each particle. The second map gives a picture of the surface LDOS $\rho_{\|}^{\text {single }}=\rho_{x}^{\text {single }}$ $+\rho_{y}^{\text {single }}$ revealing the photonic surface states (Fig. 2(C)). Above each particle, this quantity gives rise to a truncated conical protrusion with a small depression on the apex. Similar LDOS signatures have been experimentally observed when imaging gold pads with the circularly polarized tip of a near-field optical microscope. ${ }^{10}$ The last map (Fig. 2(D)) shows up the partial $z$-LDOS variation. It represents the difference between maps (B) and (C). The spatial confinement observed on these images is related to evanescent states generated by each individual particles. These particular states exist only in the near-field zone where they are tailored by the nanostructures themselves.

For more complex systems, for example, for PNN similar to those shown in Figs. 1 and 3, it is necessary to numerically solve Dyson's Eq. (2) for a system of $N$ particles placed in interaction with a surface, and then to substitute the numerical data obtained for $\mathcal{S}\left(\mathbf{r}, \mathbf{r}^{\prime}, \omega\right)$ into Eq. (1). Different information can be extracted from the numerical LDOS $\rho^{p n n}(\mathbf{r}, \omega)$ associated with a PNN adsorbed on a surface: (i) 


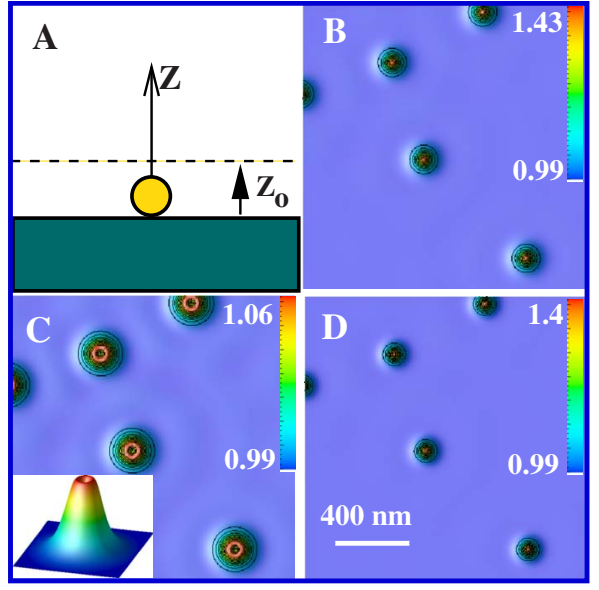

FIG. 2. (Color online) LDOS maps computed near gold nanoparticles (30 $\mathrm{nm}$ in diameter) randomly deposited on a glass surface. The operating wavelength is $530 \mathrm{~nm}$ and all the data are normalized with respect to the LDOS computed in the absence of any particle. (A) Side view of a single gold sphere supported by a planar sample (the dashed line is used to define the observation plane height); (B) total LDOS map $\rho\left(x, y, Z_{0}, \omega\right)$ computed in the plane $Z_{0}=60 \mathrm{~nm} ;(\mathrm{C})$ surface LDOS map $\rho_{x}\left(x, y, Z_{0}, \omega\right)+\rho_{y}\left(x, y, Z_{0}, \omega\right)$ calculated for $Z_{0}=60 \mathrm{~nm}$ (Inset: perspective view computed near a single particle); (D) same as (C) but for the partial LDOS along the $O Z$ direction $\rho_{z}\left(x, y, Z_{0}, \omega\right)$.

local LDOS spectra computed in predefined locations and (ii) LDOS maps that provide, for a given energy (wavelength), the topography of localized states around the PNN. In Fig. 3 we consider a typical planar PNN formed with monoparticle chains of gold beads in the vicinity of which three particular sites have been defined. In the (A) site, we have computed the total LDOS spectrum at the fixed dis-

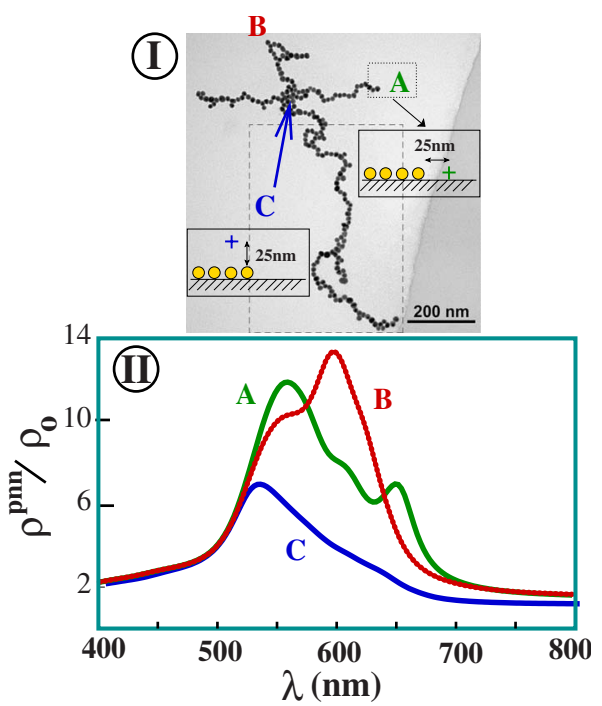

FIG. 3. (Color online) (I) TEM image of a planar PNN composed of monoparticle chains of $13 \mathrm{~nm}$ diameter gold beads deposited on a glass surface. Three different sites, labeled (A), (B), and (C), have been selected for the LDOS computation. (II) Spectra of the normalized total LDOS computed at these three sites (A), (B), and $(\mathrm{C})$.
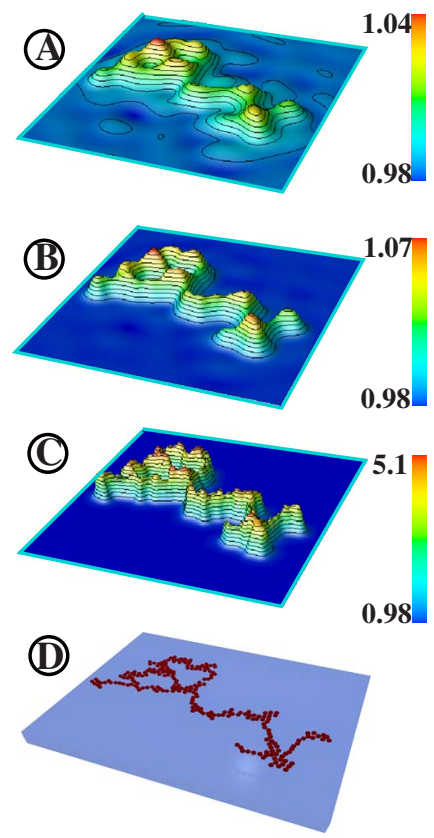

FIG. 4. (Color online) Spatial variation in the LDOS map computed over the network of Fig. 1. The maps have been computed in three consecutive planes $(Z=$ constant $)$ parallel to the sample: (A) $Z=110 \mathrm{~nm}$, (B) $Z=70 \mathrm{~nm},(\mathrm{C}) Z=32 \mathrm{~nm}$, and (D) geometry of the sample. The wavelength is $530 \mathrm{~nm}$ and the data are normalized with respect to the local density of states computed in the absence of any metal chain.

tances $D_{A}=25 \mathrm{~nm}$ from the nearest PNN bead, and $Z_{A}$ $=6.5 \mathrm{~nm}$ from the glass surface (see green curve in Fig. 3). We observe that significant LDOS enhancement occurs in a wavelength range $(500<\lambda<700 \mathrm{~nm})$. The dipolar resonance from the single (nearest) particle $(\lambda \approx 530 \mathrm{~nm})$ but also the longitudinal $(\lambda \approx 650 \mathrm{~nm})$ plasmon modes of the coupled particle chains are clearly visible. Similar spectroscopic features can be observed in (B) site (see red curve in Fig. 3) where well-aligned chain segments tend to reinforce the effect of the longitudinal plasmon mode. The (C) site, located just above the PNN node, gives rise to a drastic change in the signature because it does not allow an efficient longitudinal mode excitation to be achieved. In this particular location, the LDOS spectrum mainly reveals the individual response of the self-assembled beads (occurring around $530 \mathrm{~nm}$ ). To summarize, this analysis clearly evidences that the LDOS is significantly enhanced near the chain extremities but remains largely reinforced over all the network. This second aspect is well illustrated in Fig. 4 where three LDOS maps have been computed at the wavelength $\lambda=530 \mathrm{~nm}$ in three planes parallel to the sample depicted in Fig. 1. The color scales in this Fig. 4 indicate the variation range of the dimensionless normalized function $\rho^{p n n}(\mathbf{r}, \omega) / \bar{\rho}(z, \omega)$. The first image (4A) begins in the mesoscopic range with a map computed at $110 \mathrm{~nm}$ from the particle centers. In such a distance range, the LDOS variation is weak and the PNN is surrounded by pseudoperiodic ripples (similar to Friedel oscillations at the surface of metal) that reveal the first nonevanescent states lying around the PNN. The transition between mesoscopic and nanoscopic regime is 
visualized in the maps of Figs. 4(B) and 4(C) in which the same data are computed when decreasing the observation distance at $70 \mathrm{~nm}$ and $32 \mathrm{~nm}$ above the metal particles centers, respectively. In Fig. 4(C), we observe ripples decreasing together with a significant enhancement of the normalized LDOS in the immediate vicinity of the metal particles (see figure scale bars) that faithfully follows the different PNN branches. In this case, entering the subwavelength range dramatically increases the access to the high density of the evanescent optical modes that tend to be localized around the individual spherical particles of the PNN. At still shorter distance, for example, at $25 \mathrm{~nm}$, we have obtained a LDOS enhancement factor of 15 near PNN's adsorbed on dielectric surface. Let us note that this reinforcement of the LDOS still occurs outside the molecular quenching zone. ${ }^{29}$

To summarize, we have described a versatile formalism well suited for the analytical description and the accurate computation of the local density of photonic states in the vicinity of complex plasmonic self-assembled systems. When applied to plasmonic nanoparticle networks, this new framework shows that such systems represent unique objects for the subwavelength patterning of initially flat photonic LDOS, then revealing interesting energy transport and new photonic properties for nanoscale optics developments. In particular, the exploitation of the enhanced spectral signatures near chain extremities could lead to efficient single molecule emitters. Finally, this numerical tool can also be used to support current experimental work developed in the field of molecular plasmonics. In particular, it could be applied to other complex geometrical configurations associated with bottom-up plasmonic system deposited near surface or embedded in multilayer surroundings.
${ }^{1}$ R. Quidant et al., Europhys. Lett. 57, 191 (2002).

${ }^{2}$ M. Law et al., Science 305, 1269 (2004).

${ }^{3}$ R. M. Dickson and L. A. Lyon, J. Phys. Chem. B 104, 6095 (2000).

${ }^{4}$ J. C. Weeber, J. R. Krenn, A. Dereux, B. Lamprecht, Y. Lacroute, and J. P. Goudonnet, Phys. Rev. B 64, 045411 (2001).

${ }^{5}$ S. A. Maier et al., Adv. Mater. 13, 1501 (2001).

${ }^{6}$ H. Ditlbacher et al., Appl. Phys. Lett. 81, 1762 (2002).

${ }^{7}$ S. A. Maier, P. G. Kik, and H. A. Atwater, Appl. Phys. Lett. 81, 1714 (2002).

${ }^{8}$ A. W. Sanders et al., Nano Lett. 6, 1822 (2006).

${ }^{9}$ L. Douillard et al., Nano Lett. 8, 935 (2008).

${ }^{10}$ C. Girard and E. Dujardin, J. Opt. A, Pure Appl. Opt. 8, S73 (2006).

${ }^{11}$ K. Imura, T. Nagahara, and H. Okamoto, J. Phys. Chem. B 108, 16344 (2004).

${ }^{12}$ K. Imura, T. Nagahara, and H. Okamoto, J. Am. Chem. Soc. 126, 12730 (2004).

${ }^{13}$ C. Girard et al., Europhys. Lett. 68, 797 (2004).

${ }^{14}$ G. Colas des Francs, C. Girard, J. C. Weeber, C. Chicane, T. David, A. Dereux, and D. Peyrade, Phys. Rev. Lett. 86, 4950 (2001).

${ }^{15}$ Y. Babayan et al., ACS Nano 3, 615 (2009).
${ }^{16}$ K. Imura, T. Nagahara, and H. Okamoto, J. Chem. Phys. 122, 154701 (2005).

${ }^{17}$ S. Lin et al., Adv. Mater. 17, 2553 (2005).

${ }^{18}$ H. Zhang, K. H. Fung, J. Hartmann, C. T. Chan, and D. Wang, J. Phys. Chem. C 112, 16830 (2008).

${ }^{19}$ R. Sardar and J. S. Shumaker-Parry, Nano Lett. 8, 731 (2008).

${ }^{20}$ Y. A. Vlasov, X. Z. Bo, J. C. Sturm, and D. J. Norris, Nature (London) 414, 289 (2001).

${ }^{21}$ C. L. Haynes and R. P. V. Duyne, J. Phys. Chem. B 105, 5599 (2001).

${ }^{22}$ F. Bonell et al., J. Chem. Phys. 130, 034702 (2009).

${ }^{23}$ J. Beermann, S. I. Bozhevolnyi, and V. Coello, Phys. Rev. B 73, 115408 (2006)

${ }^{24}$ W. L. Barnes, J. Mod. Opt. 45, 661 (1998).

${ }^{25}$ G. Lévêque, G. Colas des Francs, C. Girard, J. C. Weeber, C. Meier, C. Robilliard, R. Mathevet, and J. Weiner, Phys. Rev. E 65, 036701 (2002).

${ }^{26}$ J. Nelayah et al., Nat. Phys. 3, 348 (2007).

${ }^{27}$ F. J. García de Abajo and M. Kociak, Phys. Rev. Lett. 100, 106804 (2008).

${ }^{28}$ C. Girard, Rep. Prog. Phys. 68, 1883 (2005).

${ }^{29}$ G. Baffou, C. Girard, E. Dujardin, G. Colas des Francs, and O. J. F. Martin Phys. Rev. B 77, 121101(R) (2008). 